\title{
Anisotropy measurements in mesoscopic magnets by magneto-optical torque
}

\author{
G. Armelles ${ }^{\text {a) }}$ and J. L. Costa-Krämer \\ Instituto de Microelectrónica de Madrid CSIC-CNM, Isaac Newton 8, P.T.M. Tres Cantos, \\ 28760 Madrid, Spain \\ J. I. Martin ${ }^{\text {b) }}$ \\ Dept. Física de Materiales, CC Físicas, Univ. Complutense, 28040 Madrid, Spain \\ J. V. Anguita \\ Instituto de Microelectrónica de Madrid CSIC-CNM, Isaac Newton 8, P.T.M. Tres Cantos, \\ 28760 Madrid, Spain \\ J. L. Vicent \\ Dept. Física de Materiales, CC Físicas, Univ. Complutense, 28040 Madrid, Spain
}

(Received 8 May 2000; accepted for publication 18 July 2000)

\begin{abstract}
The development of a versatile tool to quantify magnetic anisotropies in continuous and patterned magnetic thin films is reported. The technique involves measuring the magnetooptic response to a rotating magnetic field. Similarly to mechanical torque techniques, a single measurement obtains both the anisotropy constants and their symmetry axes distribution. The technique has been applied to analyze arrays of submicrometer stripe-shaped $\mathrm{Fe}(001)$ elements with different interelement separations $(s)$. For $s$ larger than $1 \mu \mathrm{m}$, the anisotropy associated with the stripes is independent on separation, with a value of the effective uniaxial anisotropy constant very consistent with theoretical estimations for these systems. (C) 2000 American Institute of Physics. [S0003-6951(00)05637-0]
\end{abstract}

The improvement in thin film growth conditions and lithographic techniques is producing a great demand to characterize in fast and straightforward fashion small patterned magnetic structures. Ordered arrays of submicrometric magnetic elements are good candidates for different memory and storage applications, due to their reduced size and controlled geometry. These structures are important as well from the basic point of view, to understand the change in magnetic properties as the size is reduced down to nanometric scales. Both theoretical and experimental approaches are then needed in order to tailor magnetic responses from the bulk material to match specific applications. These studies have just recently become possible, since the development of several patterning methods, such as electron beam ${ }^{1,2}$ or $\mathrm{x}$-ray lithography, ${ }^{3}$ has allowed the fabrication of a wide variety of patterned submicrometric magnetic structures with well defined shape, crystallinity, and geometrical distribution. ${ }^{4-6}$

On the other hand, their reduced dimensions often imply that the measurements of fundamental magnetic properties cannot be performed easily using conventional bulk techniques, requiring some important modifications or new methods. In particular, specifically designed tools are needed to measure effective magnetic anisotropy constants in patterned arrays. We present in this work a technique that obtains anisotropy constants and their symmetry axes in ordered magnetic structures of nanometric size. The power of the method is illustrated with a continuous single crystal iron film and is subsequently applied to arrays of single crystal Fe stripes.

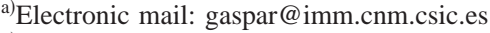

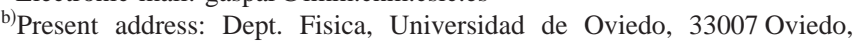
Spain.
}

The samples used in the present study are 30-nm-thick single crystal Fe (001) films grown on GaAs (001) substrates. The Fe (001) layers are covered in situ with a 2.5nm-thick capping Pt layer to avoid material oxidation. The magneto-optical hysteresis loops of these iron samples exhibit a well defined cubic biaxial anisotropy (easy axis along [100] directions) as expected. A single domain state is obtained at remanence in all the applied field directions. The arrays of patterned elements have been fabricated from the continuous $\mathrm{Fe}$ (001) films by electron beam lithography as described earlier. ${ }^{7}$ We prepare several arrays of striped shaped objects, $900 \mathrm{~nm}$ in width $(w)$ and $500 \mu \mathrm{m}$ in length $(l)$, with different stripe separation $(s=0.5,1.0$, and $1.5 \mu \mathrm{m})$. The total patterned area is a square of $500 \times 500 \mu \mathrm{m}^{2}$. Two sets of arrays have been fabricated: the first one with the stripes oriented parallel to the crystalline Fe [100] direction (magnetic easy axis); and the second with the stripes parallel to the $\mathrm{Fe}[110]$ direction (hard magnetic axis).

The magnetic anisotropy of the samples has been analyzed in a specifically designed magneto-optical torque (MOT) system that measures the changes in transverse Kerr effect signal under a rotating magnetic field. This field produces a torque in the magnetization in a similar way to mechanical torquemetry. It is also analogous to the optical technique that uses longitudinal Kerr effect to study the anisotropy in ultrathin films. ${ }^{8}$ The MOT measurements are carried out in the experimental setup sketched in Fig. 1. A magnet is mounted on a rotation stage driven by an electrical motor. The frequency of the rotation can be changed between 10 and $40 \mathrm{~Hz}$. A light source is focused on the sample. When measuring patterned structures, a light spot of less than about $100 \mu \mathrm{m}$ diameter is focused within the patterned 


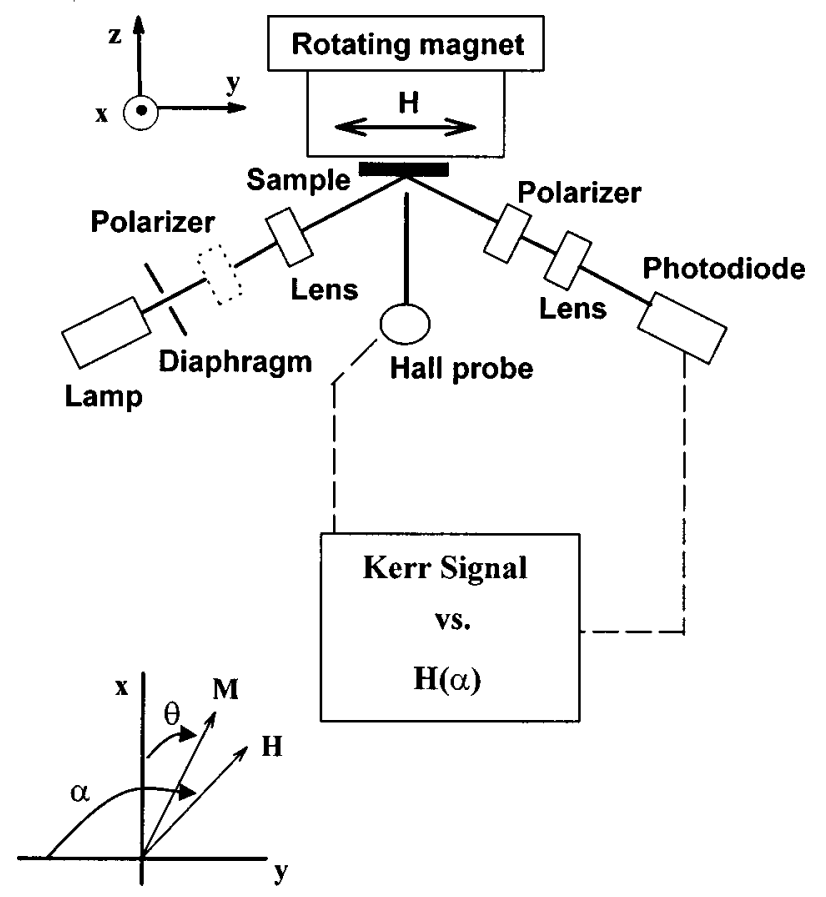

FIG. 1. Sketch (vertical view) of the magneto-optical experimental setup used to quantify magnetic anisotropies in planar film structures. Note in the insets the definition of the angles $\alpha$ and $\theta$ with respect to the vertical $x$ axis and the horizontal $y$ axis, both in the film $x y$ plane.

area. The reflected $p$-polarized light is focused on a Si photodiode. The signal from the photodiode is compensated from the direct-current (dc) offset and amplified. The measured reflectivity signal is periodic, with a period that coincides with the rotating magnet. The angle of the applied rotating magnetic field with the plane of incidence $(\alpha)$ is monitored with a Hall probe.

When the magnetic field $H$ is applied in the plane of the sample, and its magnitude is high enough to guarantee a single domain state in every direction, the dependence of the reflectivity on the direction of the applied magnetic field $(\alpha)$ can be expressed, up to second order terms in the magnetization, as $R(\alpha)=R_{0}+A \cos \theta+B \cos ^{2} \theta{ }^{9}$, where $\theta$ is the angle between the saturation magnetization and the axis perpendicular to the plane of incidence. $A$ and $B$ are coefficients which depend on the wavelength and the angle of incidence, ${ }^{10}$ for non-normal incidence $B$ is much smaller than $A$ (in our case $B$ is typically $1 \%-5 \%$ of $A$ ). This expression is derived from the reflection coefficient $r_{p p}$ which quantifies the amplitude of the reflected $p$-polarized light into $p$-polarized light and is valid only when both the incident and reflected light are $p$ polarized. If either the incident or the reflected light are unpolarized, other terms, coming from the $r_{s p}$ or $r_{p s}$ reflection coefficients may also contribute to the reflectivity. ${ }^{11,12}$ We have checked that those terms are in our case negligible by measuring the field direction dependencies of the reflectivity with and without polarized incident light, being the obtained curves in both cases the same. Therefore, the evolution of $\Delta R=R(\alpha)-R_{0}$ versus $\alpha$ represents a direct measurement of the changes in the direction of the magnetization as the magnetic field rotates.

In Fig. 2 we present the dependence of the reflectivity, for a continuous Fe film, on the angle $\alpha$, for different values of the applied field magnitude $H$. One of the easy axis is set Downloaded 08 Mar 2010 to 161.111.180.191. Redistribution subje

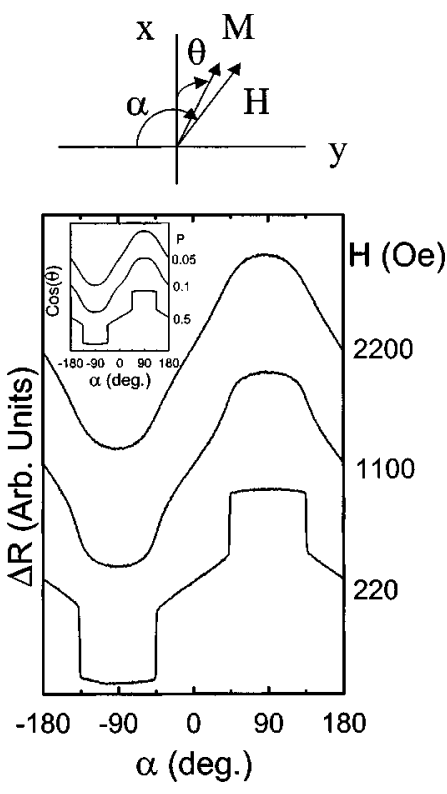

FIG. 2. Angular dependence of the reflectivity in a Fe (001) continuous film for different $H$ field magnitude values. Inset shows the calculation of the angular dependence of the magnetization projection along the $x$ axis for different values of the parameter $P=K_{c} /\left(2 H M_{S}\right)$.

perpendicular to the plane of incidence, parallel to the $x$ axis. As we increase the magnetic field amplitude the shape of the signal is strongly modified. The observed changes reflect the variation of the angle between the applied field $H$ and the saturation magnetization ("phase difference"). For a single domain system, this angle can be calculated by minimizing the magnetic free energy, which for the system sketched in Fig. 2 can be expressed as

$$
E=\left(K_{c} / 4\right) \sin ^{2} 2(\theta-\beta)=-H M_{S} \cos \left(\alpha-\theta-90^{\circ}\right),
$$

where $K_{c}$ is the anisotropy constant, $M_{S}$ the saturation magnetization, $H$ the applied field, and $\beta$ is the angle between the easy axis and the $x$ axis. For the system depicted in Fig. 2, $\beta=0^{\circ}$. In Fig. 2 (inset) we present the projection of the magnetization along the $x$ axis $(\cos \theta)$ as a function of the angle $\alpha$, for different values of $P$, where $P=K_{c} /\left(2 H M_{S}\right)$. The parameter $P$ determines the phase difference between the field and the magnetization. There are two marked regions; the first corresponds to the angular range where the magnetic field is rotating from an easy axis towards a hard axis. In this region the magnetization lags behind the field. The other region corresponds to the field rotating from the hard axis towards the easy axis. In this range the magnetization leads ahead the field. The abrupt jumps observed at $-135^{\circ},-45^{\circ}, 45^{\circ}$, and $135^{\circ}$ correspond to the transitions from one region to the other, i.e., the positions where the applied magnetic field is parallel to a hard magnetic axis. The shape of the curves is determined by the ratio $K_{c} / 2 H M_{S}$. In this Fe single crystal film, the cubic anisotropy constant obtained from a data fit to the above expressions is $K_{c}=4.1 \times 10^{5} \mathrm{erg} / \mathrm{cm}^{3}$, which agrees with the values derived from the anisotropy field of conventional hysteresis loops with $H$ applied along a hard magnetic axis. It is also similar to values reported for Fe films of comparable thickness. ${ }^{13}$

After studying the MOT response for different continuous biaxial and uniaxial systems we proceed to study systems where the method has a great advantage: its suitability to AIP license or copyright; see http://apl.aip.org/apl/copyright.jsp 


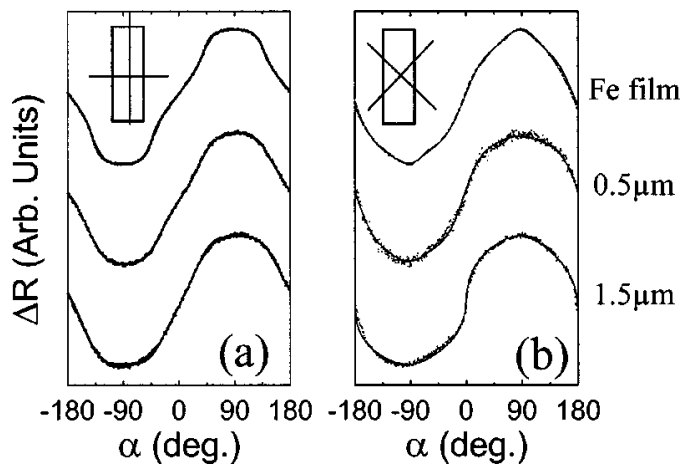

FIG. 3. Angular dependence of the reflectivity (points) and corresponding fits (lines) to Eq. (2) for arrays of Fe (001) stripes with different separation $s$ values at $H=1500 \mathrm{Oe}$. The continuous iron film data are also shown. The insets are sketches of the geometrical configurations of the stripes with respect to the crystalline Fe [100] magnetic easy axes. (a) Stripes oriented parallel to an easy [100] axis; (b) stripes oriented along a hard [110] axis.

to study the influence on the effective magnetic anisotropy of reduced dimensions and interactions in patterned submicrometric elements. As an example we present a study on arrays of single crystalline Fe stripes.

In Fig. 3 we present the MOT response for arrays of single crystalline Fe stripes. Figure 3(a) shows the data when the stripes are patterned oriented along the Fe [100] easy axis, whereas Fig. 3(b) corresponds to stripes patterned parallel to a hard [110] axis. For clarity only the curves of $s$ $=0.5$ and $1.5 \mu \mathrm{m}$ arrays are presented, together with the corresponding curves for the continuous Fe film. Note again that in Fig. 3(a) the Fe easy axis is parallel to the $x$ axis, whereas in Fig. 3(b) it is at $45^{\circ}$ with respect to the same axis, i.e., the stripes are placed in the setup vertically (parallel to the $x$ axis) in both cases. As observed, the shape of the MOT curves for the stripes is different from that for the Fe continuous film, and furthermore, there is a noticeable change as the distance between stripes, $s$, varies. These changes are due to the stripe shape anisotropy. To quantify this additional shape anisotropy we fit the data by minimizing the magnetic energy of the system. In addition to the crystalline anisotropy, we introduced now a term for the stripe shape anisotropy, that as a first order approximation can be treated as a uniaxial anisotropy. The expression in Eq. (1) for Fe continuous films is then modified for the stripes as

$$
\begin{aligned}
E= & K_{\mathrm{sh}} \sin ^{2} \theta+\left(K_{c} / 4\right) \sin ^{2} 2(\theta-\beta) \\
& -H M \cos \left(\alpha-\theta-90^{\circ}\right) .
\end{aligned}
$$

The first term corresponds to the additional uniaxial anisotropy induced by the stripes shape. The parameter $\beta$ is $0^{\circ}$ and $45^{\circ}$ for stripes parallel to easy and hard axis, respectively. The phase difference between the field and the magnetization depends now on two parameters, $P_{1}=K_{\mathrm{sh}} /\left(H M_{S}\right)$ and $P_{2}$ $=K_{c} /\left(2 H M_{S}\right)$. The value of $P_{2}$ is obtained from the continuous Fe film MOT curves fit, and is a fixed value for the theoretical fit of the MOT data for the stripes. The main results are: for arrays of stripes with $s \geqslant 1 \mu \mathrm{m}$, our data analysis shows a separation independent behavior of the effective shape anisotropy constant, with $K_{\mathrm{sh}}=5.5$ $\times 10^{5} \mathrm{erg} / \mathrm{cm}^{3}$. This value is very similar for stripes either parallel to easy or hard magnetic axes of the continuous film. For separations smaller than $1 \mu \mathrm{m} K_{\text {sh }}$ decreases significantly down to $K_{\mathrm{sh}}=2.2 \times 10^{5} \mathrm{erg} / \mathrm{cm}^{3}$ for $s=0.5 \mu \mathrm{m}$. This suggests that magnetic interactions between stripes become relevant at this small separation range, in good agreement with previous results in arrays of Fe (001) microtiles where magnetic interactions are important for interelement separations smaller than $0.9 \mu \mathrm{m}^{7}$

Our experimental values of the effective anisotropy constant associated to the stripe shape $K_{\mathrm{sh}}$ can be compared with estimations of the shape anisotropy constant $K_{\mathrm{s}}$. In these structures, it can be approximated as $K_{\mathrm{s}}=1 / 2\left(N_{\mathrm{a}}-N_{\mathrm{b}}\right) M_{\mathrm{s}}^{2}$, where $N_{\mathrm{a}}$ and $N_{\mathrm{b}}$ are the demagnetizing factors along the width and the length of the stripe, respectively, with $M_{\mathrm{s}}$ the value for the saturation magnetization of Fe. ${ }^{14}$ Considering the expressions for the demagnetizing factor of a general ellipsoid, ${ }^{15} N_{\mathrm{a}}$ and $N_{\mathrm{b}}$ for a stripe can be evaluated as $N_{\mathrm{a}}$ $=4 \pi t /(w+t)=0.13 \pi$ and $N_{\mathrm{b}}=0$. This estimation then obtains $K_{\mathrm{s}}=6.0 \times 10^{5} \mathrm{erg} / \mathrm{cm}^{3}$, which is in good agreement with our experimental result $K_{\mathrm{sh}}=5.5 \times 10^{5} \mathrm{erg} / \mathrm{cm}^{3}$.

In summary, a magneto-optical torque technique has been developed in order to characterize the magnetic anisotropy of patterned submicrometric structures. The method has been applied to arrays of Fe (001) stripes with different interelement separations $s$. The effective shape anisotropy induced by the patterning is independent of the separation distance $(s)$ for $s \geqslant 1 \mu \mathrm{m}$, with $K_{\mathrm{sh}}=5.5 \times 10^{5} \mathrm{erg} / \mathrm{cm}^{3}$, in excellent agreement with shape anisotropy estimations. For the smallest separation this effective anisotropy is reduced down to $2.2 \times 10^{5} \mathrm{erg} / \mathrm{cm}^{3}$, due to the presence of magnetic interactions between neighbors elements.

Work supported by the Spanish CICYT and Comunidad de Madrid. The authors wish to thank A. Cebollada and J. C. Martinez for providing with the samples used in this work and F. Briones and H. Rubio for useful discussions.

${ }^{1}$ J. F. Smyth, S. Scultz, D. R. Fredkin, D. P. Ken, S. A. Rishton, H. Schmid, M. Cali, and T. R. Koehler, J. Appl. Phys. 69, 5262 (1991).

${ }^{2}$ S. Y. Chou, P. R. Kraus, and L. Kong, J. Appl. Phys. 79, 6101 (1996).

${ }^{3}$ M. Hehn, K. Ounadjela, J. P. Bucher, F. Rousseaux, D. Decanini, B. Bartenlian, and C. Chappert, Science 272, 1782 (1996).

${ }^{4}$ C. Shearwood, S. J. Blundell, M. J. Baird, J. A. C. Bland, M. Gester, H. Ahmed, and H. P. Hughes, J. Appl. Phys. 75, 5249 (1994).

${ }^{5}$ J. Yu, U. Rüdiger, L. Thomas, S. S. P. Parkin, and A. D. Kent, J. Appl. Phys. 85, 5501 (1999).

${ }^{6}$ J. I. Martín, Y. Jaccard, A. Hoffmann, J. Nogués, J. M. George, J. L. Vicent, and I. K. Schuller, J. Appl. Phys. 84, 411 (1998).

${ }^{7}$ J. L. Costa-Krämer, J. I. Martín, J. L. Menéndez, A. Cebollada, J. V. Anguita, F. Briones, and J. L. Vicent, Appl. Phys. Lett. 76, 3091 (2000).

${ }^{8}$ R. Mattheis and G. Quednau, J. Magn. Magn. Mater. 205, 143 (1999).

${ }^{9}$ M. J. Freisner, IEEE Trans. Magn. 4, 152 (1968).

${ }^{10}$ G. A. Bolotin, Fiz. Met. Metalloved. 39, 731 (1975).

${ }^{11}$ R. M. Osgood III, S. D. Bader, B. M. Clemens, R. L. White, and H. Matsuyama, J. Magn. Magn. Mater. 182, 297 (1998).

${ }^{12}$ R. M. Osgood III, B. M. Clemens, and R. L. White, Phys. Rev. B 55, 8990 (1997).

${ }^{13}$ R. J. Hicken, S. J. Gray, A. Ercole, C. Daboo, D. J. Freeland, E. Gu, E. Ahmad, and J. A. C. Bland, Phys. Rev. B 55, 5898 (1997).

${ }^{14}$ B. D. Cullity, Introduction to Magnetic Materials (Addison-Wesley, Reading MA, 1972), p. 243

${ }^{15}$ J. A. Osborn, Phys. Rev. 67, 351 (1945). 